\title{
Development of Rapid Synchrotron 2D X-ray Mapping and XANES of Rock Slabs and Drill Core as a Tool for the Mineral Exploration and Mining Industries.
}

\author{
Neil R. Banerjee $^{1 *}$, Lisa L. Van Loon ${ }^{1,2}$, Trevor J. Flynn ${ }^{1}$ and David Muir ${ }^{3}$ \\ 1. Department of Earth Sciences, Western University, London, Canada. \\ 2. LISA CAN Analytical Solutions Inc, Saskatoon, Canada. \\ 3. Canadian Light Source, Saskatoon, Canada \\ * Corresponding author, neil.banerjee@uwo.ca
}

Mineral exploration is a pillar of Canada's economy with annual mineral production topping $\$ 45$ billion a year, creating $>375,000$ jobs, $\$ 89.5$ billion in exports, more than $\$ 677$ million in $\mathrm{R} \& \mathrm{D}$ expenditures and a better standard of living for all Canadians. New mineral deposit discoveries require novel methodologies and approaches to detect large-scale ore-forming systems and to vector into their highgrade cores. Synchrotron science is an innovation, presently under-utilized by the broader geosciences community, with the potential to change the way in which rapid, cost effective, and high fidelity techniques are used to address industry relevant questions. But the barrier to adoption of these techniques by the broader geoscience community is significant. Through a unique collaboration between academia, industry, and synchrotrons we are tackling this problem head on to develop techniques for the mineral exploration and mining industries.

Synchrotron X-ray fluorescence (SR-XRF) mapping provides rapid and cost-effective micron-scale trace element analysis and mapping of ore minerals with ppm detection limits. Speciation of trace elements important for understanding geometallurgy and element mobility can be probed using X-ray absorption near-edge structure (XANES) spectroscopy. For example, SR-XRF mapping and XANES analysis of pyrite grains associated with gold deposits in several world-class gold camps in Canada's north have revealed key indicators of mineralization history and novel trace element exploration vectors. Gold in these deposits is present both as free and/or "invisible" gold, bound in the pyrite crystal lattice. The high flux and energy of a synchrotron allows for in situ and non-destructive detection of invisible gold by $\mu \mathrm{XRF}$ and probes its nature (metallic $\mathrm{Au}^{0}$ vs. lattice bound $\mathrm{Au}^{+1}$ ) using XANES spectroscopy.

Experimental: Rock slabs and drill core were analyzed by SR-XRF and XANES at the IDEAS Beamline at the Canadian Light Source (CLS) in Saskatoon, SK, to create 2D trace element maps that reveal spatial and structural associations with gold mineralization. The incident X-ray energy was set to $13750 \mathrm{eV}$. A Ketek ${ }^{\circledR}$ AXAS-M single element SDD was used to measure the XRF signal.

SR-XRF mapping was done by rastering across the sample with beam spot sizes of $500 \mu \mathrm{m} \times 500 \mu \mathrm{m}, 1$ $\mathrm{mm} \times 1 \mathrm{~mm}$, or $2 \mathrm{~mm} \times 2 \mathrm{~mm}$ across the sample, in both directions and a dwell time of 1 second. The distance between the sample and the detector was kept constant for all samples. SR-XRF data analysis was done with Peakaboo 4 [1]. Spot As K-edge and Au L 3 -edge XANES spectra were collected in fluorescence mode with the samples positioned in front of the X-ray beam at a $45^{\circ}$ angle. The detector was windowed to correspond to the $\mathrm{As} \mathrm{K} \alpha$ or $\mathrm{Au} \mathrm{L} \alpha$ fluorescence emission peak to maximize the signal to noise. XANES data was collected using a beam spot size of $500 \mu \mathrm{m}$ x $500 \mu \mathrm{m}$ to $2 \mathrm{~mm} \times 2 \mathrm{~mm}$. Data were collected over an energy range of $-100 \mathrm{eV}$ to $+100 \mathrm{eV}$ (relative to the edge), with a dwell time of 2 sec per point and a step size of $1 \mathrm{eV}$ near the edge. Two scans were collected per spot. XANES data were processed using Athena [2]. 
Results: We made several key technological innovations driven by frequent and collaborative interaction with our industry partners and beamline staff. (1) Mineral exploration and mining companies want to be able to analyze cores exactly as it is laid out in their core sheds so that the geochemical data produced can be immediately related to the core in context. We built custom designed sample holders for large and heavy rock core and slab samples and overlaid the chemical maps on top of high quality digital photomicrographs. (2) Companies also want to be able to map much larger areas so that the mineralogical and structural context available during core logging is preserved. We increased the mapping area at other beamlines by three orders of magnitude to $>4000 \mathrm{~mm}^{2}(4 \mathrm{~cm} \mathrm{x} 10 \mathrm{~cm})$. The area of the XRF map is now exactly matched to industry standard core. An example of SR-XRF single element mapping of a rock slab can be seen in Figure 1. (3) Companies wanted the XRF mapping to rapid to survey more core samples. We increased the beam size incrementally from $<10$ microns to $>2$ $\mathrm{mm}$ and created maps at several resolutions to determine the optimal beam size. Using a larger beam size improved throughput by a factor of $>10$ without compromising sensitivity and with the added benefit of lower detection limits due to the larger number of photons interacting with the sample.

Summary: Three main gold distributions have been identified: free gold, gold metal in sulphide phases (likely micro/nano scale particles), and gold in the crystal lattice of arsenic rich sulphides (both arsenian pyrite and arsenopyrite). Structural features are frequently visible in the XRF maps, which is helpful in correlating both elemental and timing relationships of the gold mineralization. The XRF maps also reveal the association of gold with various major and trace elements useful as exploration vectors. XANES spectroscopy can distinguish between metallic nano-inclusions and lattice bound refractory gold in sulphide minerals, which has important implications for economic recovery. XANES spectroscopy is also an excellent tool for determining speciation of any deleterious elements identified during XRF mapping. This innovative, multi-faceted approach provides insights into fluid evolution, mineralization conditions, controls on mineralization and gold remobilization, the economics of recovery, and environmental considerations that can be applied to myriad deposit types.

Figure. 1. Example of a rock slab photograph followed by single element 2D SR-XRF maps of Fe, As, and Ca collected with a beam spot size of $1 \mathrm{~mm} \times 1 \mathrm{~mm}$. Several veins and structures visible in the photo are seen in the element maps.

\section{References:}

[1] N. Sherry et al, Science Studio Project Summary. (2012) http://sciencestudio.net/summary.php.

[2] B. Ravel, M. Newville. J. Synchr. Rad. 12 (2005) p. 537.

[3] Research described in this paper was performed at the Canadian Light Source, which is supported by the CFI, NSERC, the Univ. of Saskatchewan, the Government of Saskatchewan, Western Economic Diversification Canada, the NRC, and the CIHR. The authors acknowledge funding from NSERC, TerraX Minerals Inc., and Yamana Gold Inc.

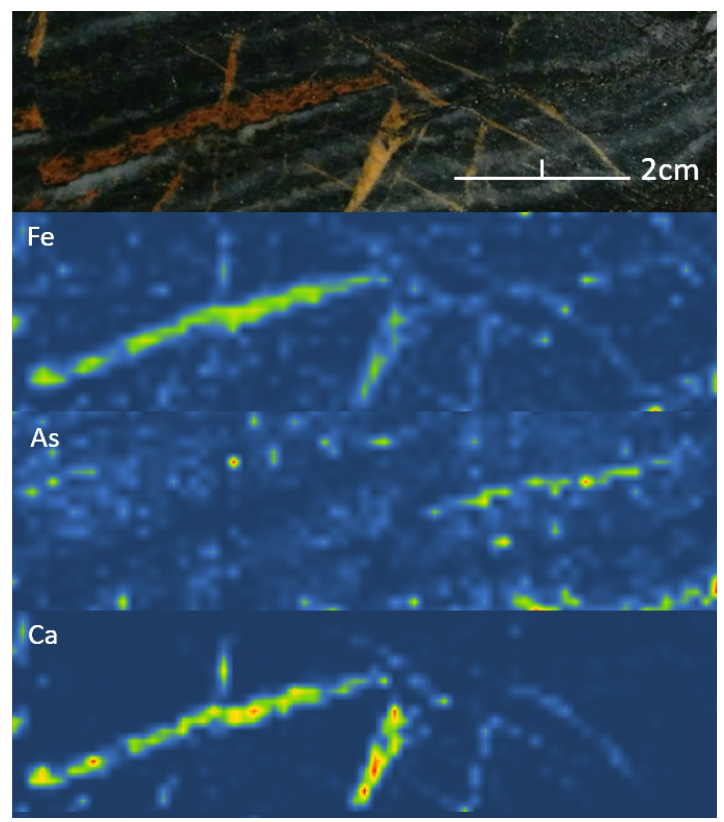

